\title{
A DIFFERENTIATION THEOREM FOR FUNCTIONS DEFINED ON THE DYADIC RATIONALS
}

\author{
R. J. LINDAHL
}

AbSTRACT. In this paper we show that under certain conditions a real-valued function defined on an interval of dyadic rational numbers is a monotone function. One of these conditions involves a generalized differentiability property. From this result we offer a new proof of a conjecture of $\mathrm{N}$. Fine concerning the uniqueness of solution of Walsh series.

1. Introduction. Let $f$ be a real-valued function defined on $(a, b)$ $\cap D, a<b$, where $D$ denotes the set of dyadic rational numbers. In this paper we shall describe conditions on $f$ which will ensure that $f$ is monotone decreasing on $(a, b) \cap D$. This will enable us to reprove a conjecture of N. Fine [2] concerning the uniqueness of solution of Walsh series.

For our purposes we introduce the following functions.

Given any nonnegative integer $n$ and real number $x$, let $\alpha_{n}(x)$ $=k / 2^{n}, \beta_{n}(x)=(k+1) / 2^{n}$ where $k$ is that integer for which $k \leqq 2^{n} x<k$ +1 . Also set

$$
\begin{aligned}
\alpha_{n}^{\prime}(x) & =\alpha_{n}(x), & & x \notin D, \\
& =\alpha_{n}(x)-1 / 2^{n}, & & x \in D .
\end{aligned}
$$

Now given any $x$ in $(a, b)$ we write

$$
D_{-} f(x)=\liminf _{n \rightarrow \infty}\left[f\left(\beta_{n}(x)\right)-f\left(\alpha_{n}(x)\right)\right] \cdot 2^{n} .
$$

2. The main theorem. Let $G$ be a real-valued function defined on $(a, b) \cap D$. Our primary result can be stated as follows.

THEOREM. Assume that $G$ satisfies the following conditions:

(i) $\lim \sup _{n \rightarrow \infty} G\left(\alpha_{n}^{\prime}(x)\right) \geqq G(x), x \in(a, b) \cap D$.

(ii) $\liminf _{n \rightarrow \infty}\left[G\left(\beta_{n}(x)\right)-G\left(\alpha_{n}(x)\right)\right] \leqq 0, x \in(a, b)$.

(iii) $D \_G(x) \leqq 0, x \in(a, b) \backslash E$ for some countable set $E$.

Then $G$ is monotone decreasing on $(a, b) \cap D$.

Proof. Clearly one may assume that $E=\left\{x_{k}\right\}_{k=1}^{\infty}$ contains the

Received by the editors June 10, 1969 and, in revised form, November 9, 1970.

AMS 1969 subject classifications. Primary 2640, 2650; Secondary 4211.

Key words and phrases. Monotone functions on dyadic rationals, Walsh series, Walsh-Fourier series. 
dyadic rationals in $(a, b)$. Moreover, by considering the functions $G_{\epsilon}(x)=G(x)-\epsilon x, x \in(a, b)$, for each $\epsilon>0$ we see that one may assume $G$ satisfies

$$
\liminf _{n \rightarrow \infty} 2^{n}\left[G\left(\beta_{n}(x)\right)-G\left(\alpha_{n}(x)\right)\right]<-\epsilon
$$

for some $\epsilon>0$ and all $x$ in $(a, b) \backslash E$. Now once we have established that

$$
G\left(k / 2^{p}\right) \leqq G\left((k-1) / 2^{p}\right), \quad(k-1) / 2^{p}, k / 2^{p} \in(a, b),
$$

where $k$ and $p$ are integers, $p \geqq 0$, the theorem follows. Hence assume to the contrary that there exists integers $k_{0}$ and $p, p \geqq 0$, with $\left(k_{0}-1\right) / 2^{p}, k_{0} / 2^{p} \in(a, b)$ such that

$$
G\left(k_{0} / 2^{p}\right)>G\left(\left(k_{0}-1\right) / 2^{p}\right) .
$$

Set $\xi_{0}=\left(k_{0}-1\right) / 2^{p}, \eta_{0}=k_{0} / 2^{p}, I_{0}=\left[\xi_{0}, \eta_{0}\right]$. It will prove convenient to define a set function $\mu$ by:

$$
\mu([\xi, \eta])=G(\eta)-G(\xi), \quad \xi, \eta \in D \cap(a, b) .
$$

Then $\mu\left(I_{0}\right)>0$ by assumption. Let $E_{0}=E$ and define $I_{n}, E_{n}$ inductively by the following procedure. Having chosen $I_{n}=\left[\xi_{n}, \eta_{n}\right]$, $\xi_{n}, \eta_{n} \in(a, b) \cap D$ with $\mu\left(I_{n}\right)>0$ and $E_{n}=E \cap I_{n}$, let

$$
I_{n}^{1}=\left[\xi_{n}, \frac{\xi_{n}+\eta_{n}}{2}\right], \quad I_{n}^{2}=\left[\frac{\xi_{n}+\eta_{n}}{2}, \eta_{n}\right] .
$$

If $E_{n}$ is empty, set $I_{n+1}=I_{n}^{i}$ where $i \in\{1,2\}$ is smallest possible such that $\mu\left(I_{n}^{i}\right)>0$. If $E_{n}$ is nonempty, let $n^{\prime}$ be the smallest subscript such that $x_{n}$ is in $E_{n}$. Assume first that $x_{n^{\prime}}$ is the midpoint of $I_{n}$. Then set $I_{n+1}=I_{n}^{i}$ where $i \in\{1,2\}$ is smallest possible such that $\mu\left(I_{n}^{i}\right)>0$. If $x_{n^{\prime}}$ is not the midpoint of $I_{n}$, one has $x_{n^{\prime}} \in I_{n}^{i}, x_{n^{\prime}} \in I_{n}^{j},\{i, j\}=\{1,2\}$. Set $I_{n+1}=I_{n}^{j}$ if $\mu\left(I_{n}^{j}\right)>0$. Otherwise set $I_{n+1}=I_{n}^{i}$. This defines $I_{n+1}$ $=\left[\xi_{n+1}, \eta_{n+1}\right], \xi_{n+1}, \eta_{n+1} \in(a, b) \cap D$. Set $E_{n+1}=E \cap I_{n+1}$. In each of the above cases we have $\mu\left(I_{n+1}\right)>0$ since $\mu\left(I_{n}\right)=\mu\left(I_{n}^{1}\right)+\mu\left(I_{n}^{2}\right)>0$.

Observe that for each $n, I_{n}$ has length $2^{-(p+n)}$. Moreover if $I_{n+1}=I_{n}^{i}$ and $\mu\left(I_{n}^{j}\right) \leqq 0$, then $\mu\left(I_{n+1}\right) \geqq \mu\left(I_{n}\right)$ where $\{i, j\}=\{1,2\}$. Set $\{x\}$ $=\bigcap_{n=0}^{\infty} I_{n}$ and notice that $\bigcap_{n=1}^{\infty} E_{n}=E \cap\{x\}$.

Case 1. $x \in E \cap D$. Then there exists $N>0$ such that for each $n>N, x$ is the left (or right) endpoint of $I_{n}$. Suppose that $x$ is the left endpoint for $n>N$. Then $I_{n}=\left[\alpha_{n+p}(x), \beta_{n+p}(x)\right], I_{n}=I_{n-1}^{1}, \mu\left(I_{n-1}^{2}\right) \leqq 0$. Hence $\mu\left(I_{n-1}\right) \leqq \mu\left(I_{n}\right)$ for all $n>N$ which implies that

$$
\underset{n \rightarrow \infty}{\liminf }\left[G\left(\beta_{n}(x)\right)-G\left(\alpha_{n}(x)\right)\right]>0 .
$$


This contradicts (ii). Assume next that $x$ is a right endpoint for $n>N$. Again one has $\mu\left(I_{n-1}\right) \leqq \mu\left(I_{n}\right)$ and $I_{n}=\left[\alpha^{\prime}{ }_{n+p}(x), x\right]$ so

$$
\liminf _{n \rightarrow \infty}\left[G(x)-G\left(\alpha_{n}^{\prime}(x)\right)\right]>0
$$

which contradicts (i).

Case 2. $x \in E, x \in D$. Then for each $n, I_{n}=\left[\alpha_{n+p}(x), \beta_{n+p}(x)\right]$ and $\mu\left(I_{n}\right) \leqq \mu\left(I_{n+1}\right)$ which again contradicts (ii).

Case 3. $x \notin E$. As in Case 2 we can write $I_{n}=\left[\alpha_{n+p}(x), \beta_{n+p}(x)\right]$ for all $n$. Consequently,

$$
\liminf _{n \rightarrow \infty} 2^{n}\left[G\left(\beta_{n}(x)\right)-G\left(\alpha_{n}(x)\right)\right] \geqq 0
$$

which contradicts (iv). This completes the proof.

It should be remarked that this result improves the lemma of $\mathrm{N}$. Fine as given in [2, p. 407].

3. An application to Walsh series. In 1947, N. Fine in his classical paper on Walsh-Fourier series [2] considered the problem of determining when a Walsh series is the Walsh-Fourier series of a Lebesgue integrable function. It was conjectured that given a Walsh series which converges to an integrable function except on a countable set, the series is the Walsh-Fourier series of the function. This conjecture was proved in 1964 by R. Crittenden and V. Shapiro in [1]. Their proof was rather lengthy and involved an intricate application of the Baire category theorem. We offer a simplified proof using only the results in [2] together with the main theorem.

We now introduce some standard terminology and restate certain theorems from [2] which will be used in the sequel. Let $\psi_{k}$ denote the $k$ th Walsh function on the interval $[0,1]$ and set $J_{k}(x)=\int_{0}^{x} \psi_{k}(t) d t$, $x \in[0,1]$, for $k=0,1, \cdots$.

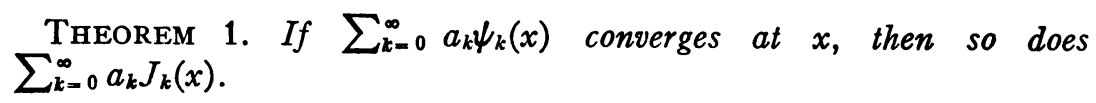

From $[2$, p. 405] one has with slight modifications:

THEOREM 2. If $\left(a_{k}\right)_{k=0}^{\infty}$ converges to zero and $L(x) \equiv \sum_{k=0}^{\infty} a_{k} J_{k}(x)$ defines an essentially absolutely continuous function on $[0,1]$, then $\sum_{k=0}^{\infty} a_{k} \psi_{k}(x)$ is the Walsh-Fourier series of $L^{\prime}(x)$.

From the two theorems in $[2$, p. 406] one has:

THEOREM 3. Let $\left(a_{k}\right)_{k=0}^{\infty}$ converge to zero, set $L(x) \equiv \sum_{k=0}^{\infty} a_{k} J_{k}(x)$, $x \in[0,1]$. Then this series converges for each $x \in D \cap[0,1]$. Moreover for 
each $x \in[0,1], L\left(\beta_{n}(x)\right)-L\left(\alpha_{n}(x)\right)=2^{-n} S_{2^{n}}(x)$ which converges to zero uniformly in $x$.

From these results together with the main theorem we now prove the conjecture.

THEOREM 4. Let $\sum_{k=0}^{\infty} a_{k} \psi_{k}(x)$ converge to a finite-valued integrable function $f$ except on a countable set of points $E$ in $[0,1]$. Then this series is the Walsh-Fourier series of $f$.

Proof. Set $F(x)=\int_{0}^{x} f(t) d t, x \in[0,1]$, and fix $\epsilon>0$. By the VitaliCarathéodory theorem [3, p. 75] one can select two absolutely continuous functions $\phi_{\epsilon}$ and $\psi_{\epsilon}$ on $[0,1]$ such that

$$
\left|\phi_{\epsilon}(x)-F(x)\right|<\epsilon, \quad\left|\psi_{\epsilon}(x)-F(x)\right|<\epsilon, \quad x \in[0,1],
$$

and the derivates of $\phi_{\epsilon}(x)$ (resp. $\psi_{\epsilon}(x)$ ) are less than (resp. greater than) $f(x)$ whenever $f(x) \neq-\infty$ (resp. $f(x) \neq+\infty)$. For $x$ in $D \cap(0,1)$, set $G_{\epsilon}(x)=\phi_{\epsilon}(x)-L(x), H_{\epsilon}(x)=L(x)-\psi_{\epsilon}(x)$. The functions $G_{\epsilon}$ and $H_{\epsilon}$ exist by Theorem 3. By Theorem $3, G_{\epsilon}$ and $H_{\epsilon}$ satisfy the hypothesis in the lemma. Hence $G_{\epsilon}$ and $H_{\epsilon}$ are monotone decreasing on $D \cap(0,1)$. Letting $\epsilon$ tend to zero one has that $F-L$ and $L-F$ are monotone decreasing on $(0,1) \cap D$. Hence $F-L$ is constant on $(0,1) \cap D$ so setting $L(x)=F(x)+c, x \in(0,1) \cap D$, we see that this equality extends by Theorem 3 to all $x$ for which $L(x)$ exists. By Theorem 1, $L(x)$ exists for all $x \in E$. Consequently, $L$ is essentially absolutely continuous on $[0,1]$ so, by Theorem $2, \sum_{k=0}^{\infty} a_{k} \psi_{k}(x)$ is the WalshFourier series of $L^{\prime}(x)=f(x)$.

\section{BIBLIOGRAPHY}

1. R. B. Crittenden and V. L. Shapiro, Sets of uniqueness on the group $2^{\omega}$, Ann. of Math. (2) 81 (1965), 550-564. MR 31 \#3783.

2. N. J. Fine, On the Walsh functions, Trans. Amer. Math. Soc. 65 (1949), 372414. MR 11, 352.

3. S. Saks, Théorie de l'intégrale, Monografie Mat., vol. 2, PWN, Warsaw, 1933; English transl., Monografie Mat., vol. 7, PWN, Warsaw; Hafner, New York, 1937.

Pennsylvania State University, University Park, Pennsylvania 16802 